\title{
STAR results on local strong P/CP violation at $\mathrm{RHIC}$
}

\author{
Evan Finch* \\ Yale University \\ E-mail: evan.finch@yale.edu \\ for the STAR Collaboration
}

\begin{abstract}
It has been proposed for some time now that local parity violation may occur in heavy-ion collisions. Recent theoretical work has suggested a mechanism by which this would lead to electromagnetic charge separation along the collision angular momentum vector. We review STAR measurements of a correlator which is directly sensitive to this effect and shows a significant signal in $\mathrm{Au}+\mathrm{Au}$ and $\mathrm{Cu}+\mathrm{Cu} 200 \mathrm{GeV}$ collisions which agrees qualitatively with many of the features of the proposed parity violation. This correlator is parity even and is therefore susceptible to various backgrounds; we discuss and show event-generator model estimates for these.
\end{abstract}

5th International Workshop on Critical Point and Onset of Deconfinement

June 8-12, 2009

Brookhaven National Laboratory, Long Island, New York, USA

\footnotetext{
* Speaker.
} 


\section{Introduction}

Current understanding of QCD tells us that violation of parity (P) and CP symmetries by the strong interaction is permitted. The size of the violation is governed by the parameter $\bar{\Theta}$, an angular variable which contains contributions both from the structure of the QCD vacuum (which is necessary to explain the large $\eta^{\prime}$ mass) and also (basically unrelated contributions) from the quark mass matrix; $\bar{\Theta}$ is naturally expected to be of order 1 [1]. Given this, it is startling that $\bar{\Theta}$ is experimentally constrained to be $\lesssim 10^{-10}$ [2]; the cause of this is unknown and this is commonly referred to as the Strong CP problem.

Among the motivations for pursuing the experimental study of heavy-ion collisions is the hope of finding measurable consequences of exciting the QCD vacuum state [3]. It has been proposed that among these consequences may be the formation of metastable states in heavy-ion collisions in which the excited vacuum breaks $\mathrm{P}$ and $\mathrm{CP}$ symmetries $[4,5,6]$ to a large degree (effectively, with $\bar{\Theta}$ of order one). Continued work on this topic has led to a proposal for a specific way in which this violation may manifest itself, called the "Chiral Magnetic Effect" (CME)[7, 8].

In the CME, the enormous (electromagnetic) magnetic field of the heavy ion collision causes quark spins to tend to become oriented along (or directly against, depending on the charge) the collision angular momentum vector. The region in which parity violation occurs produces a nonzero chirality of (assumed massless) quarks, which is understood to happen by changes to the momenta of quarks in the region. Ultimately, this leads to a separation of charge along the angular momentum vector. Whether the positive quarks in one particular parity violating region move in the direction of $\vec{L}$ or opposite to it depends on the properties of the excited vacuum in the metastable region (in particular a property of the fields called the "topological charge") and is effectively random in each created region. Whichever way the positive charges move, the negative charges move in the opposite direction. The final effect that we will look for experimentally is clear: a separation of electromagnetic charge along the angular momentum vector.

\section{Method}

We look at the azimuthal distribution of particles in an event

$$
\frac{d N_{ \pm}}{d \phi} \propto\left(1+2 v_{1} \cos (\Delta \phi)+2 v_{2} \cos (2 \Delta \phi)+\ldots+a_{ \pm} \sin (\Delta \phi)+\ldots\right),
$$

and if such a local parity violation occurs we would expect a non-zero $a_{-}=-a_{+}\left(v_{1}\right.$ and $v_{2}$ give the strength of "directed" and "elliptic" flow). Because the relative direction of the angular momentum vector and charge separation should vary event-to-event, we expect that averaged over many events, $\left\langle a_{+}\right\rangle=\left\langle a_{-}\right\rangle=0$. (To verify this, we need to know the first order reaction plane in each event. Using the STAR ZDC Shower Max Detector, we have checked this and found that for minimum bias 200 $\mathrm{GeV} \mathrm{Au}+\mathrm{Au}$ collisions $\left\langle a_{+}\right\rangle,\left\langle a_{-}\right\rangle \lesssim 10^{-4}$ ).

If $\langle a\rangle$ must be zero, what can we measure? Clearly, the CME will cause an increase in fluctuations of (for example) the number of charged particles going in the direction of the magnetic field. We turn, then, to fluctuation/correlation measurements; specifically, measurements of $\left\langle a_{+} a_{+}\right\rangle,\left\langle a_{-} a_{-}\right\rangle$, and $\left\langle a_{+} a_{-}\right\rangle$. We may think of $\left(a_{+} a_{+}\right)$as the correlation of two values of $a_{+}$ 
determined from different parts of the same event and $\left\langle a_{+} a_{+}\right\rangle$as this value averaged over many events. If there were no other correlations within an event and the correlations from the CME were preserved throughout the evolution of the system, then this local parity violation would lead to values $\left\langle a_{+} a_{+}\right\rangle=\left\langle a_{-} a_{-}\right\rangle=-\left\langle a_{+} a_{-}\right\rangle>0$.

These correlations are parity even quantities and so measurements of them are generally plagued by various backgrounds. An observable proposed in [9] which avoids many of these is

$$
\begin{aligned}
\left\langle\cos \left(\phi_{\alpha}+\phi_{\beta}-2 \Psi_{R P}\right)\right\rangle= & \left\langle\cos \Delta \phi_{\alpha} \cos \Delta \phi_{\beta}\right\rangle-\left\langle\sin \Delta \phi_{\alpha} \sin \Delta \phi_{\beta}\right\rangle \\
& =\left[\left\langle v_{1, \alpha} v_{1, \beta}\right\rangle+B_{\text {in }}\right]-\left[\left\langle a_{\alpha} a_{\beta}\right\rangle+B_{\text {out }}\right] .
\end{aligned}
$$

with $\Delta \phi=\left(\phi-\Psi_{R P}\right)$ being the azimuthal angle of a given particle relative to the reaction plane. Here, $\alpha$ and $\beta$ represent electric charge + or - and the average is taken over all pairs in an event and then over all events (of a given centrality class). $\left\langle a_{\alpha} a_{\beta}\right\rangle$ is the quantity we wish to measure. In the results presented in this proceedings, $\left\langle v_{1, \alpha} v_{1, \beta}\right\rangle$ is minimized in practice by analyzing particles in a symmetric rapidity region near mid-rapidity. Under the assumption that $v_{1}$ fluctuations in the region $|\eta|<1$ are not larger than $v_{1}(\eta=1)$, this contribution is negligibly small compared to the other terms here. $B_{\text {in }}\left(B_{\text {out }}\right)$ represents background contributions from any parity-respecting correlated particle production which has a non-zero projection onto (out of) the reaction plane. By using the observable $\left\langle\cos \left(\phi_{\alpha}+\phi_{\beta}-2 \Psi_{R P}\right)\right\rangle$, we see only the difference $B_{\text {in }}-B_{\text {out }}$ and so are only sensitive to such correlations to the extent that they have reaction plane dependence, significantly reducing the task of estimating this background which will be discussed in section 4 .

In practice, we do not know the reaction plane and must evaluate $\left\langle\cos \left(\phi_{\alpha}+\phi_{\beta}-2 \Psi_{R P}\right)\right\rangle$ using the measured event plane as $\left\langle\cos \left(\phi_{\alpha}+\phi_{\beta}-2 \Psi_{E P}\right)\right\rangle$ which is then corrected by the event plane resolution. In most of the analyses discussed here, the reaction plane is estimated by the 2nd order event plane of a class of particles labeled "c", in which case we further write

$$
\left\langle\cos \left(\phi_{a}+\phi_{\beta}-2 \Psi_{R P}\right)\right\rangle=\left\langle\cos \left(\phi_{a}+\phi_{\beta}-2 \phi_{c}\right)\right\rangle / v_{2, c}
$$

where $v_{2, c}$ describes the elliptic flow of the "c" particles. This relation is strictly true only if the particles used to find the reaction plane are correlated with the groups of particles $\alpha$ and $\beta$ only through their common correlation to the reaction plane. We will explore this assumption later in the talk. For now we will proceed to look at the STAR measurement results under the assumption that $\left\langle\cos \left(\phi_{a}+\phi_{\beta}-2 \phi_{c}\right)\right\rangle / v_{2, c}$ gives a measurement of $\left\langle a_{\alpha} a_{\beta}\right\rangle$, though we know that there are potential backgrounds which we be subsequently discussed.

\section{Results}

In the left hand panel of Fig. 1, we see the results of a STAR [10] analysis of $\left\langle\cos \left(\phi_{a}+\phi_{\beta}-\right.\right.$ $\left.\left.2 \phi_{c}\right)\right\rangle / v_{2, c}$ from RHIC $200 \mathrm{GeV} \mathrm{Au}+\mathrm{Au}$ (run 4) and $\mathrm{Cu}+\mathrm{Cu}$ (run 5) collisions. The kinematic range for included tracks is $|\eta|<1,150 \mathrm{MeV} / \mathrm{c}<p_{T}<2 \mathrm{GeV} / \mathrm{c}$ and acceptance corrections (which are small) are applied following [11]. At first glance, Fig. 1 has many of the features initially expected for the CME: the same-charge and opposite-sign correlations are both of the expected sign for all centralities in $\mathrm{CuCu}$ and many of the centralities in $\mathrm{AuAu}$ (the signal crosses to the "wrong" sign in central $\mathrm{AuAu}$ - more on this later — as can be seen in the right hand panel of Fig. 1 in which 

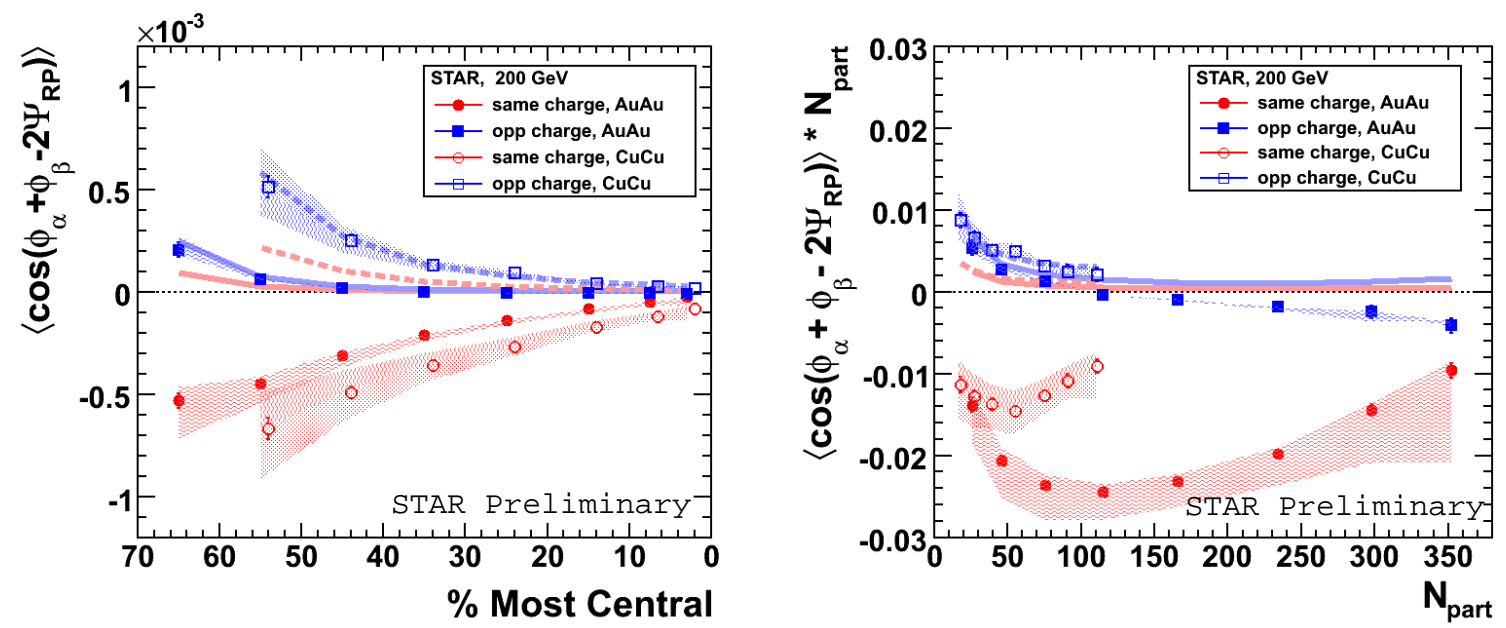

Figure 1: $\left\langle\cos \left(\phi_{a}+\phi_{\beta}-2 \Psi_{R P}\right)\right\rangle$ in $\mathrm{Au}+\mathrm{Au}$ and $\mathrm{Cu}+\mathrm{Cu}$ collisions at $\sqrt{s_{N N}}=200 \mathrm{GeV}$ as a function of centrality (central collisions to the right) with error-bars representing statistical errors. The shaded area reflects the uncertainty in the elliptic flow values used in calculations. Thick solid ( $\mathrm{Au}+\mathrm{Au})$ and dashed $(\mathrm{Cu}+\mathrm{Cu})$ lines represent non-reaction-plane-dependent background contribution from many-particle clusters as estimated by HIJING; this is discussed in the section concerning backgrounds. In the right hand plot, the observable is scaled by the estimated number of participants in each centrality bin.

$\left\langle\cos \left(\phi_{\alpha}+\phi_{\beta}-2 \Psi_{R P}\right)\right\rangle$ is scaled by the number of participants in part to make this region more visible).

The centrality dependence and overall size of the like-sign signal also are similar to the initial CME prediction [12] that $a_{\alpha}$ should be roughly $Q / N_{\pi^{+}}$where $Q$, the typical topological charge of the parity violating region, is of order 1 . The signal from the opposite-charge correlations is clearly smaller in magnitude than the like-charge signal (assuming for now that $B_{\text {in }}-B_{\text {out }}$ is small). This becomes more promising with the suggestion (made in [7] after STAR results were first shown) that the opposite-charge correlations may be suppressed by the medium (the expected opposite-charge correlation is a correlation between particles traveling in opposite directions, implying that at least one of the partners typically has a long path length to escape the medium) to a much larger degree than the like-sign correlations. Whether this mechanism can quantitatively explain the difference is a key open question for theory to answer.

In Fig. 2 we have the dependence of the correlator on the sum and difference of $\left|p_{t}\right|$ of the two particles of type $\alpha$ and $\beta$ in the 30-50\% centrality range of $\mathrm{Au}+\mathrm{Au}$ collisions. From the left hand panel, we note that the signal is roughly constant for a difference in $\left|p_{t}\right|$ from 0 to $2 \mathrm{GeV} / \mathrm{c}$ which argues strongly against the signal having its origins in femtoscopic correlations. In the right hand panel we see that the signal grows roughly linearly with $p_{t}$ up to $2 \mathrm{GeV} / \mathrm{c}$. The original expectation was that the signal should grow with $p_{t}$ at low $p_{t}$ but generally should come dominantly from the region $p_{t}<1 \mathrm{GeV} / \mathrm{c}$. Whether the observed behavior as a function of $p_{t}$ can be reconciled with more complete calculations with the Chiral Magnetic Effect is another important task for the theory. 

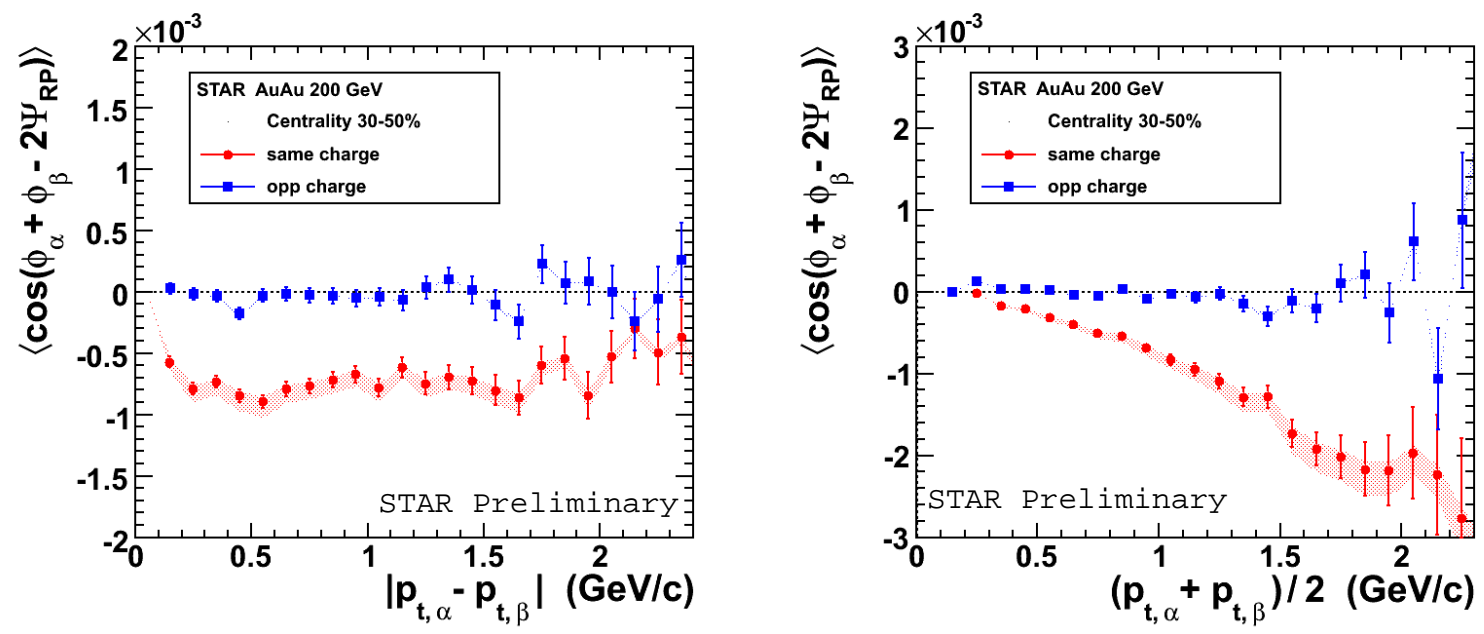

Figure 2: Dependence of the signal on $\left|p_{t, \alpha}-p_{t, \beta}\right|$ (left) and $\left(p_{t, \alpha}+p_{t, \beta}\right) / 2$ (right) in $200 \mathrm{GeV} \mathrm{Au+Au}$ collisions.

\section{Physics Backgrounds}

We now turn our attention to possible physics backgrounds. We will divide these into two categories.

1. Clusters of 3 or more particles. As noted above, if there is a correlation linking particles of type "c" to particles of types " $\alpha$ " or " $\beta$ " other than common correlation to the reaction plane, then the factorization in eq. 2.3 will not be exact. Clearly, such correlations will exist at some level for the results shown in Fig. 1 since in this case all particles used in the three-particle correlation are in the pseudorapidity region of the STAR main TPC $(|\eta|<1$.). We can estimate the size of this background using event generator models; the one that we have found to make the largest prediction for this background is HIJING [13] (with jet quenching turned off) and this is shown for $\mathrm{Au}+\mathrm{Au} 200 \mathrm{GeV}$ events in Fig. 3. If we assume the HIJING calculation of this background is correct, the remaining signal for the oppositecharge $\left\langle\cos \left(\phi_{\alpha}+\phi_{\beta}-2 \Psi_{R P}\right)\right\rangle$ becomes similar to zero (again, neglecting $B_{\text {in }}-B_{\text {out }}$ for now) in many centrality bins while the same-sign correlation is not greatly affected. UrQMD [16] gives a considerably smaller (by about a factor of three) prediction for this background but there is concern that all such models may under-predict such background as we move to central events [14].

In principle, this reaction-plane-independent background is reducible experimentally if we can measure the reaction plane in a way that has no such correlations with particles of types $\alpha$ and $\beta$. A straightforward way is to use particles that are separated in rapidity from the signal particles to find the reaction plane. For the results shown in Fig. 4 we do just this. The left panel compares results obtained using the reaction plane found in the TPC against results with the reaction plane found in the FTPC $(2.7<|\eta|<3.9)$. The results are strikingly similar, particularly for the same-charge correlation measurements which have quite small relative uncertainties over much of the centrality range. Still, we should be concerned that 


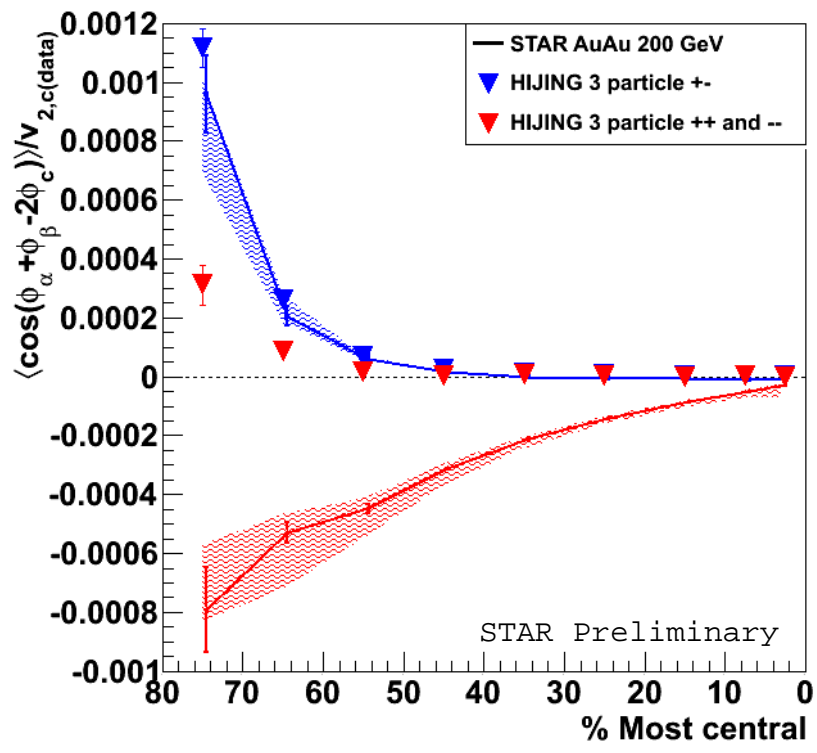

Figure 3: $\left\langle\cos \left(\phi_{a}+\phi_{\beta}-2 \phi_{c}\right)\right\rangle / v_{2, c}$ from data (lines) compared with HIJING predictions (triangles) for the reaction-plane-independent background from clusters of three or more particles.

there may be correlations that reach over this rapidity gap to connect particles found in the FTPC with TPC particles. This concern disappears if we can find the reaction plane with the STAR ZDC-SMD with which we measure the azimuthal distribution of spectator neutrons to determine the event plane. We show such a measurement in the right panel of Fig. 4. The measurements made with the ZDC-SMD event plane are consistent with the measurements using the TPC reaction plane. However, with the available 2004 data, the statistical error bars from this measurement are still too large to allow us to differentiate between 3-particle cluster contribution at the level that HIJING predicts and that which is predicted by UrQMD (or none at all).

2. Clusters of two or more particles which have reaction plane dependence $\left(B_{\text {in }}-B_{\text {out }}\right)$.

This type of background is much more insidious, as it cannot be beaten down by an improved reaction plane measurement and it is unclear to what extent it can be experimentally removed at all. We may crudely consider the observable $\left\langle\cos \left(\phi_{\alpha}+\phi_{\beta}-2 \Psi_{R P}\right)\right\rangle$ to represent [(\# of same-side in-plane pairs) - (\# of opposite-side in-plane pairs) $]-$ [(\# of same-side out-of-plane pairs) -(\# of opposite-side out-of-plane pairs)]. So we see, for example, that a resonance which decays with small opening angle (giving a "same-side" pair) will, if it exhibits a positive $v_{2}$, give a positive contribution to $\left\langle\cos \left(\phi_{\alpha}+\phi_{\beta}-2 \Psi_{R P}\right)\right\rangle$. Of course, the reaction plane dependence that leads to a contribution to this background may be not just $v_{2}$ but may include also different decay/fragmentation in-plane compared to out-of-plane.

We can estimate these contributions from known sources (including $\rho$, $\omega$, etc. resonance decays and jets which contain a charged particle of large enough momentum to provide an 

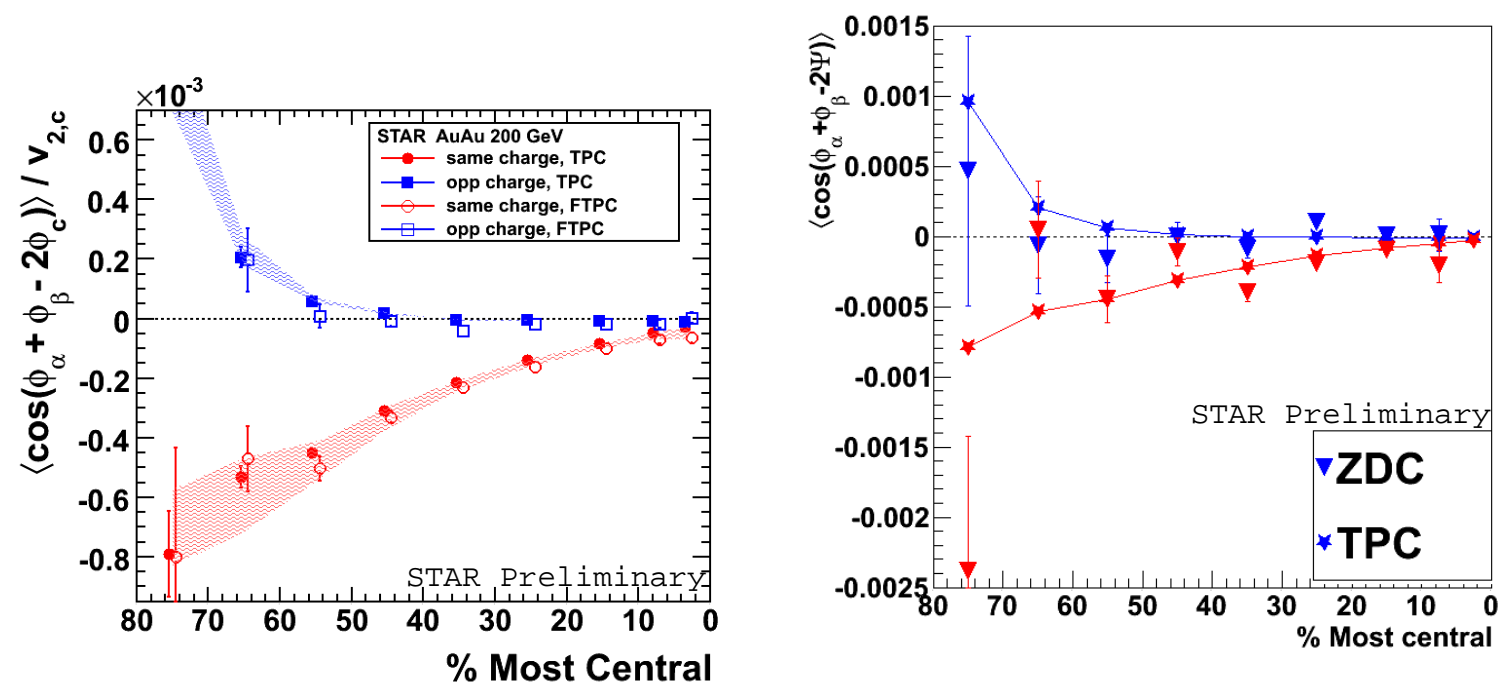

Figure 4: LEFT: Comparison of $\left\langle\cos \left(\phi_{\alpha}+\phi_{\beta}-2 \Psi_{R P}\right)\right\rangle$ calculated with event-plane "c" particles found in the TPC against that with "c" particles in the FTPC. Error bars in each case represent statistical error while the shaded bands represent the systematic uncertainty on the "TPC" measurement due to reaction plane determination. RIGHT: A similar comparison as in the LEFT panel, but now between reaction plane found in the ZDC-SMD and TPC. In this plot, only statistical errors are shown.

analysis "trigger") and find that they cannot qualitatively explain the correlations seen in Fig. 1. To search more broadly we have used various heavy ion event generators to calculate $\left\langle\cos \left(\phi_{\alpha}+\phi_{\beta}-2 \Psi_{R P}\right)\right\rangle$ (with $\Psi_{R P}$ known) in these models. We show some of these results in Fig. 5 for HIJING (also HIJING with an afterburner which adds data-like $v_{2}$ to all final-state particles), UrQMD, and MEVSIM [15]. HIJING and UrQMD, of course, are true heavy-ion event models whereas MEVSIM is a simple event generator in which the only correlations (in our use) come from resonance decays and an overall data-like flow pattern. We see that no event generator gives qualitative agreement with the observed like-charge correlation signal or with the observed separation between the like-charge and opposite-charge signal seen in data.

In principle, the reaction-plane-dependent background should be subtracted away (as should the reaction-plane-independent background) before labeling our measurement $\left\langle a_{\alpha} a_{\beta}\right\rangle$. Because of the large uncertainty in the background level, this is not done. We note, however, that if the reaction-plane-dependent-background is as large as predicted by, for example, UrQMD (as shown in Fig. 5), then we should not consider the opposite-charged measurements in central Au-Au to be of the wrong sign and in fact the magnitude of $\left\langle a_{+} a_{-}\right\rangle$is not dramatically less than the magnitude of $\left\langle a_{+} a_{+}\right\rangle,\left\langle a_{-} a_{-}\right\rangle$and rather is more line with initial theoretical (without away-side quenching) expectations that the magnitudes should be approximately equal. Clearly, an improved theoretical understanding of the reaction-planeindependent background is crucial to the interpretation of this measurement.

Other sources of background (including global hyperon polarization with respect to the re- 


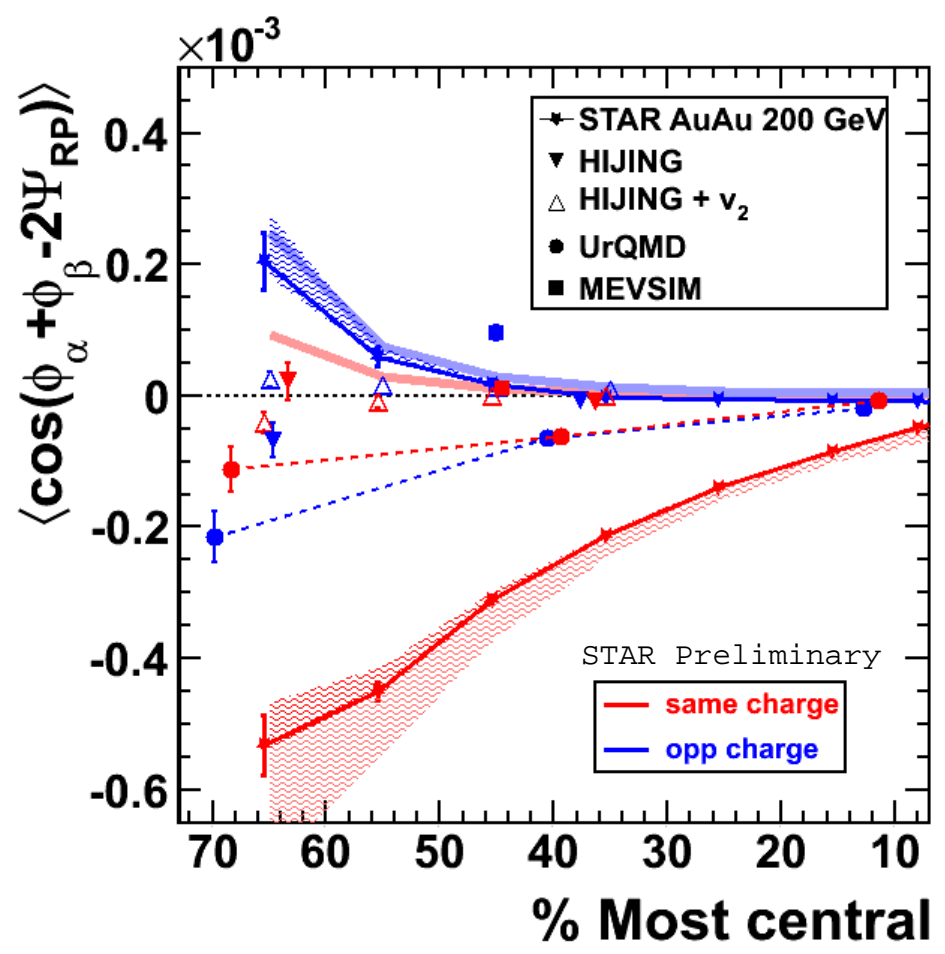

Figure 5: Lines represent calculations of $\left\langle\cos \left(\phi_{\alpha}+\phi_{\beta}-2 \Psi_{R P}\right)\right\rangle$ from data and thick lines represent three particle background from HIJING. Various symbols represent two-particle reaction plane-dependent background (i.e. $\left\langle\cos \left(\phi_{\alpha}+\phi_{\beta}-2 \Psi_{R P}\right)\right\rangle$ with $\Psi_{R P}$ assumed known from the event generator) in various model calculations.

action plane [17]) have been considered and found to be negligible compared to the size of the signal.

\section{Summary and Outlook}

We have shown the signal measured by STAR for $\left\langle\cos \left(\phi_{\alpha}+\phi_{\beta}-2 \Psi_{R P}\right)\right\rangle$ in $200 \mathrm{GeV} \mathrm{Au}+\mathrm{Au}$ and $\mathrm{Cu}+\mathrm{Cu}$ collisions. This observable is directly sensitive to $\left\langle a_{\alpha} a_{\beta}\right\rangle$ but, being parity even, is also sensitive to various backgrounds. In the studies shown here we have found no backgrounds or combination of backgrounds that can qualitatively reproduce the measured like-charge correlations or the separation seen in Fig. 1 between like-charge and opposite-charge correlations. Further study, both experimental (particularly to further constrain three-particle reaction-plane-independent backgrounds) and theoretical (to better understand reaction-plane-dependent cluster backgrounds) will be extremely important to understanding whether these correlations are truly indications of local parity violation via the Chiral Magnetic Effect.

Until now, theoretical work on the CME has concentrated on establishing the likelihood of the effect occurring in heavy ion collisions and estimating roughly the expected features of the signal coming from the CME. Clearly, it is also now important to have more quantitative predictions from the theory where possible, particularly addressing questions raised by these measurements: what is 
the expected $p_{T}$ dependence of the signal(?) and can we understand quantitatively the difference in magnitude between same- and opposite- charged correlations? In general, the effect on the expected signal of evolution of the collision system including hadronization will be of considerable interest.

There are also numerous possibilities for further experimental work to further test whether these correlations are in fact due to the CME. Measuring these correlations among different identified neutral particles which should not feel the CME (reaction plane azimuthal correlations using $\pi^{0}$ s would be a wonderful test, but probably not practical) would be an important test. In addition, one fairly firm prediction of the theory is that the magnitude of $\left\langle a_{+} a_{+}\right\rangle,\left\langle a_{-} a_{-}\right\rangle$should scale with the square of the magnitude of the magnetic field created in the collision region by the colliding ions (and therefore for fixed $A$ should scale with $Z^{2}$ ). Comparing results of collisions of isobars would thus be a very interesting test of this effect. Observing the signal as a function of collision energy in the upcoming RHIC beam energy scan will also be informative (the correlations at 62 $\mathrm{GeV}$ were not included in this proceedings, but have been measured by STAR and found to be qualitatively similar to and of slightly larger magnitude than those at $200 \mathrm{GeV}$ ). There do not yet exist quantitative predictions for the signal behavior as a function of beam energy, but without deconfinement and chiral restoration it is expected that strength of the CME will dramatically drop so we would expect the signal to eventually disappear as sufficiently low energy is reached.

That local parity violation may take place in heavy ion collisions is an extremely interesting prediction. The experimental results shown here hint that this may in fact occur and point to further work both theoretical and experimental to establish this effect.

\section{References}

[1] R. D. Peccei, Lect. Notes Phys. 741, 3 (2008).

[2] C. A. Baker et al., Phys. Rev. Lett. 97, 131801 (2006).

[3] T. D. Lee, Rev. Mod. Phys. 47, 267 (1975).

[4] T. D. Lee and G. C. Wick, Phys. Rev. D 9, 2291 (1974).

[5] P. D. Morley and I. A. Schmidt, Z. Phys. C 26, 627 (1985).

[6] D. Kharzeev, R. D. Pisarski and M. H. G. Tytgat, Phys. Rev. Lett. 81, 512 (1998).

[7] D. E. Kharzeev, L. D. McLerran and H. J. Warringa, Nucl. Phys. A 803, 227 (2008).

[8] K. Fukushima, D. E. Kharzeev and H. J. Warringa, Phys. Rev. D 78, 074033 (2008).

[9] S. A. Voloshin, Phys. Rev. C 70, 057901 (2004).

[10] K. H. Ackermann et al. [STAR Collaboration], Nucl. Instrum. Meth. A 499, 624 (2003).

[11] I. Selyuzhenkov and S. Voloshin, Phys. Rev. C 77, 034904 (2008).

[12] D. Kharzeev, Phys. Lett. B 633, 260 (2006).

[13] M. Gyulassy and X.-N. Wang, Comput. Phys. Commun. 83, 307 (1994); X.N. Wang and M. Gyulassy, Phys. Rev. D 44, 3501 (1991).

[14] M. Daugherity [STAR Collaboration], J. Phys. G 35, 104090 (2008).

[15] R. L. Ray and R. S. Longacre, arXiv:nucl-ex/0008009.

[16] S. A. Bass et al., Prog. Part. Nucl. Phys. 41, 255 (1998)

[17] B. I. Abelev et al. [STAR Collaboration], Phys. Rev. C 76, 024915 (2007). 